\title{
Nasal and orbital recurrence of conjunctival melanoma 21 years after exenteration
}

Department of Pathology, Institute of Ophthalmology A D A Paridaens A C E McCartney

Department of Ear, Nose and Throat Diseases, St Bartholomew's Hospital R J Lavelle

Department of Ophthalmology, St Bartholomew's Hospital, and Department of Oncology, Moorfields Eye Hospital $\mathrm{J}$ L Hungerford Correspondence to: Dr A D A Paridaens, Institute of Ophthalmology, Department of Pathology, 17-25 Cayton Street, London ECIV 9AT.

Accepted for publication 14 November 1991

\author{
A D A Paridaens, A C E McCartney, R J Lavelle, J L Hungerford
}

\begin{abstract}
In our experience $5 \%$ of invasive malignant melanomas of the conjunctiva arising from areas of primary acquired melanosis with atypia spread to the ipsilateral nasal cavity and paranasal sinuses. Twenty one years after orbital exenteration for multicentric conjunctival melanoma an 82-year-old man was seen with an orbital recurrence, which had extended to the nasal cavity and paranasal sinuses through the nasolacrimal duct without invading the mucosa. This previously undescribed way of spread after the longest symptom-free interval following exenteration ever reported is illustrated.
\end{abstract}

Malignant melanomas of the nasal cavity or paranasal sinuses which develop in patients with conjunctival melanoma are rare. We report on a patient who was thought to have been cured of conjunctival malignant melanoma of his right eye but presented with an unusual orbital and nasal recurrence 21 years after exenteration of his right orbit.

\section{Case report}

A 60-year-old Caucasian man was treated in 1969 by a modified anterior exenteration of the right orbit, a skin splitting technique being used, for multifocal malignant melanoma of the conjunctiva. There was extensive involvement of the caruncle and the inferior palpebral and bulbar

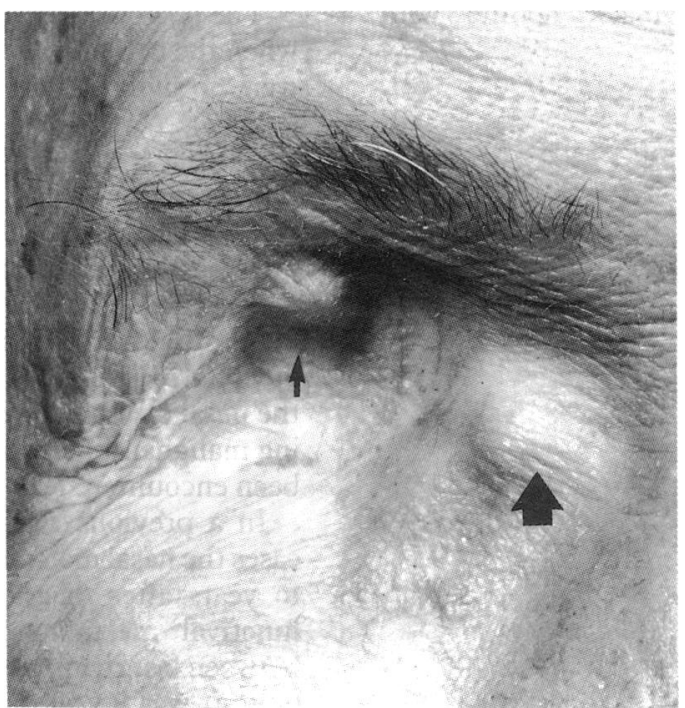

Figure 1 A large subcutaneous recurrence of conjunctival malignant melanoma (large arrow) anteromedially in the right orbit, which had been exenterated 21 years previously. The small, subcutaneous, hard and immobile superolateral nodule (small arrow) remained unchanged over several years and was not associated with the disease. conjunctiva. The exenteration specimen showed multiple foci of heavily pigmented malignant melanoma arising from areas of primary acquired melanosis with atypia. The planes of surgical excision were clear of tumour.

In 1985 a small, hard subcutaneous nodule, which was tethered to the periosteum superiorly in the right socket, was noted. The overlying skin was slightly pigmented. On observation the small mass remained unchanged.

In 1990, 21 years after the exenteration, the 82-year-old patient complained of obstruction of the right nasal passage. Examination of the right orbit showed a large subcutaneous swelling at the medial edge of the right socket and the preexisting small nodule at the superolateral edge (Fig 1). Examination of the nasal cavity revealed a large ulcerating pigmented polyp obstructing the right nasal passage (Fig 2). There was no clinical involvement of the regional lymph nodes. Investigation for metastases, including liver ultrasound, liver function tests, and a chest $x$ ray, was negative. There was no evidence of cutaneous malignant melanoma. A computerised tomography (CT) scan (Fig 3) showed the presence of tumour in the right anteromedial orbit, the nasolacrimal duct, invasion of the anterior ethmoidal cells, and a large intranasal mass. A diagnostic biopsy of the nasal lesion confirmed the clinical diagnosis of malignant melanoma. The patient's remaining eye showed lens opacities and age-related macular disease, reducing the visual acuity to $6 / 12$.

The patient was treated by right lateral rhinotomy. The skin overlying the medial orbital mass was excised. The anteromedial wall of the maxilla and the orbital floor were removed, and subsequently the tumour was cleared from the

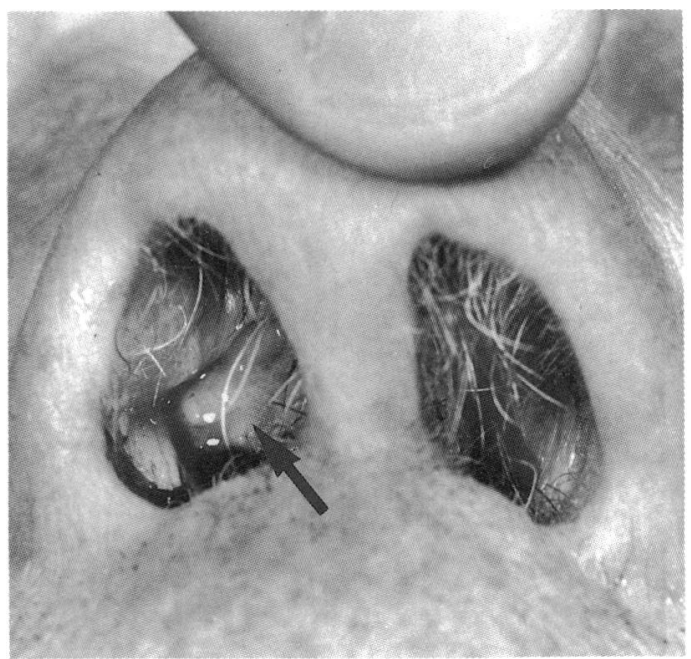

Figure 2 Ulcerating malignant melanoma (arrow) which obstructed the right nasal passage and was connected with the orbital mass through the nasolacrimal duct. 


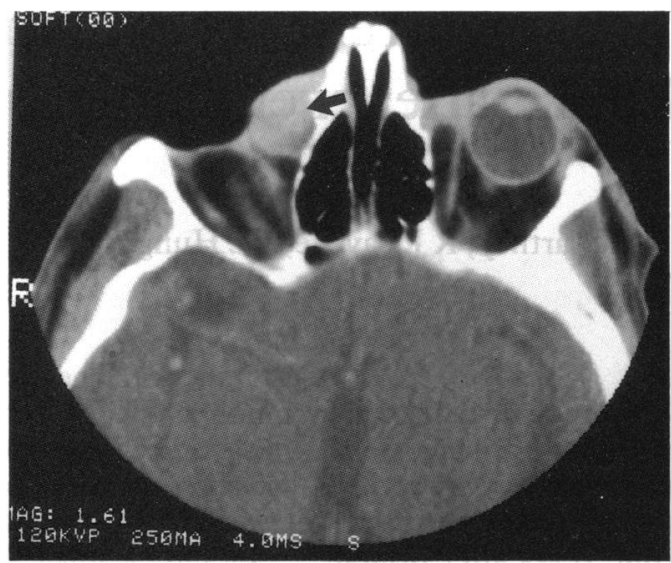

Fig $3 A$

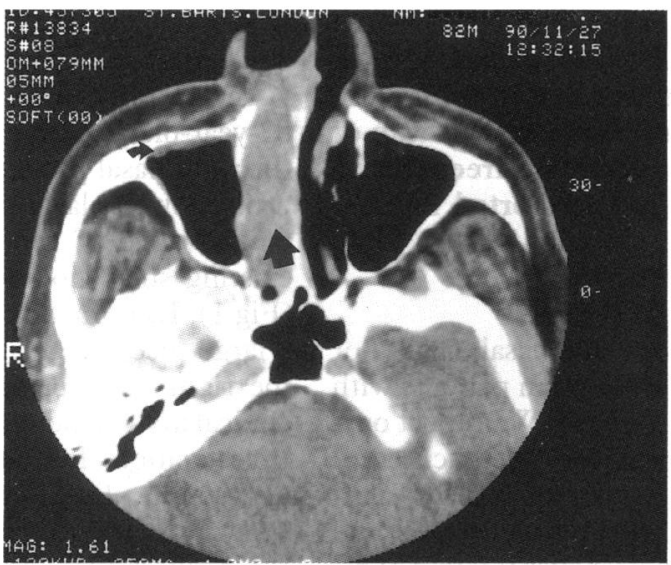

Fig $3 C$

maxillary antrum, the anterior ethmoidal cells, and the nasal cavity. At surgery it became apparent that the nasal element of the disease was a direct extension of the orbital recurrence through the nasolacrimal duct, without invading mucosa or bone. A forehead flap was used to close the orbitonasal defect.

Histological examination of the excised orbital element showed orbital skin with an underlying, well demarcated grey-black nodule with a

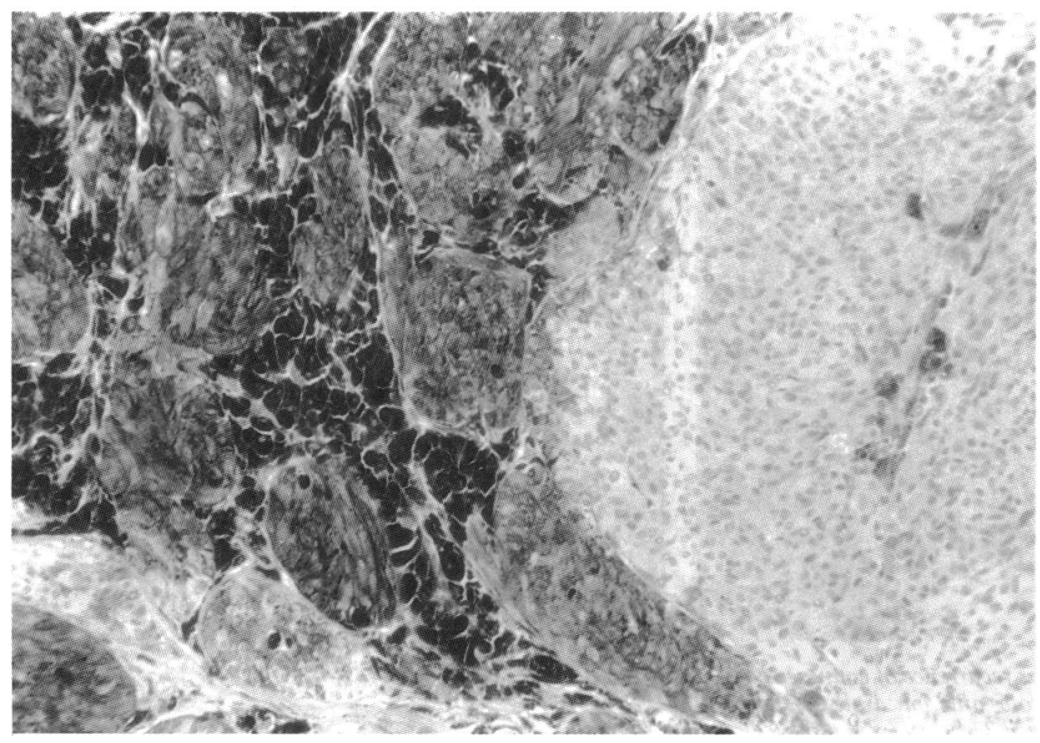

Figure 4 Histological section of the orbital subcutaneous grey-black nodule with a maximum diameter of $8 \mathrm{~mm}$, showing malignant melanoma with varying degrees of pigmentation (haematoxylin and eosin, $\times 185$ ).

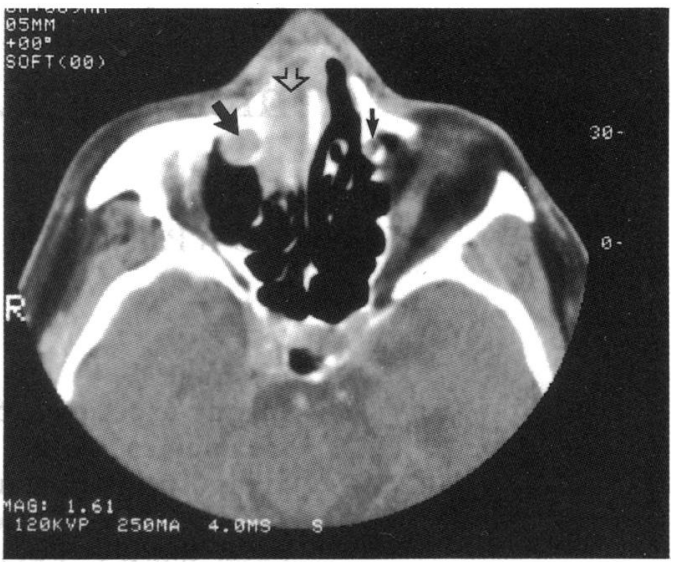

Fig $3 B$

Figure 3 Orbital axial CT scans. (A) a large soft tissue mass (arrow) lies anteromedially within the exenterated right orbit. Note the residual optic nerve and medial and lateral rectus muscles; $(B)$ the right nasolacrimal duct (large arrow) has been expanded by a soft tissue mass and is $100 \%$ larger than its normal counterpart (small arrow). There is a large intranasal mass (open arrow) with invasion of the anterior ethmoidal cells; $(C)$ the nasal soft tissue mass (large arrow) fills the entire nasal cavity with no evidence of residual conchae. The right maxillary antrum shows a thin line of mucosal thickening (curved arrow), which is probably secondary to inflammation. In this section there is no evidence of tumour in the maxillary sinus.

maximum diameter of $8 \mathrm{~mm}$, consistent with malignant melanoma (Fig 4). The degree of pigmentation varied from amelanotic to deeply pigmented. Histologically the nasal element consisted of similar pigmented tumour, which contained focal spindle areas and showed focal necrosis. Histological study of superolateral orbital skin revealed fibrosis of the dermis but no tumour.

The postoperative course was uneventful, and when the patient was last seen in June 1991 there were no signs of local or regional metastases.

\section{Discussion}

Between 1949 and 1991 more than 260 cases of histologically confirmed invasive malignant melanoma of the conjunctiva have been treated in Moorfields Eye Hospital and St Bartholomew's Hospital. Eight cases of malignant melanoma of the nasal cavity and paranasal sinuses complicating malignant melanoma of the conjunctiva have been encountered.

In a previous report' on four of these eight cases the nasal melanoma was recognised months to years after diagnosis of the ipsilateral conjunctival melanoma. The maximum interval between last treatment and diagnosis of the nasal tumour was 9 years. ${ }^{1}$ In our case the recurrence was diagnosed 21 years after orbital exenteration, which is the longest symptom-free interval reported after such treatment. This finding emphasises the importance of long follow-up periods in the study of conjunctival malignant 


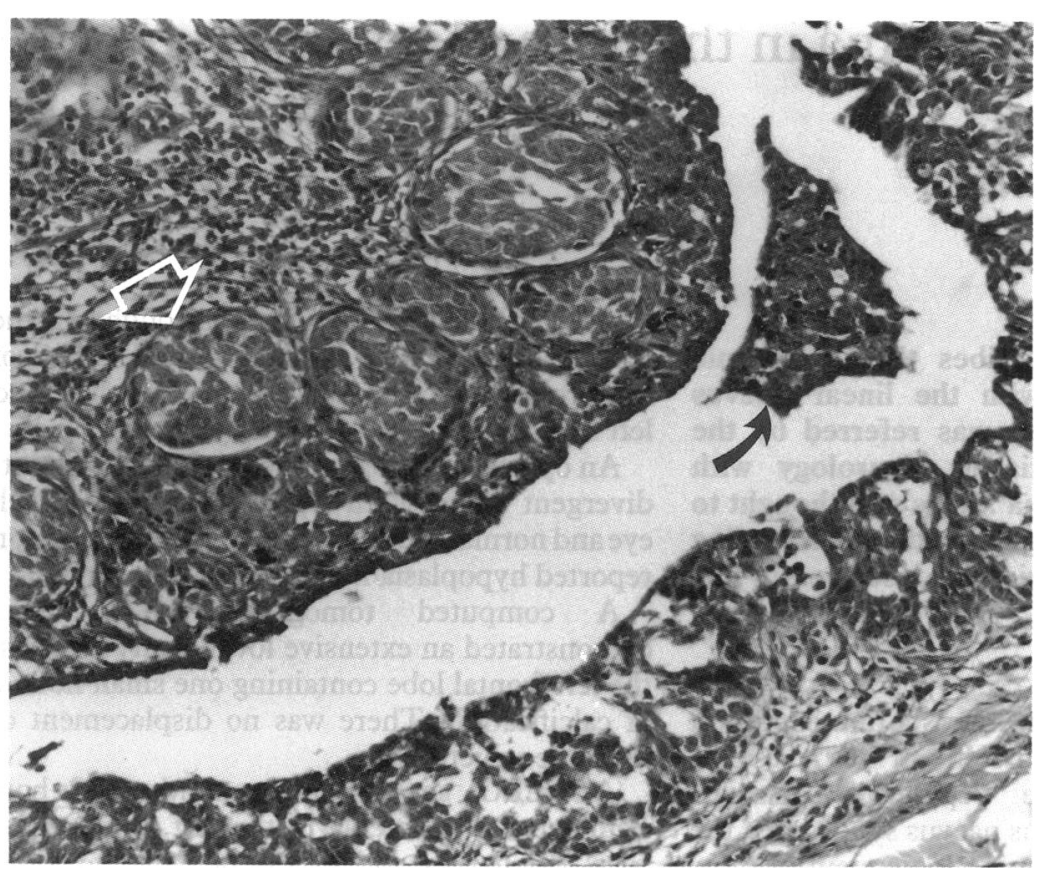

Figure 5 Malignant melanoma cells in the lumen (black arrow) and the wall (white arrow) of the lacrimal apparatus in an exenteration specimen (haematoxylin and eosin, $\times 300$ ).

melanoma. As no other foci of malignant melanoma were found in this patient, the recurrent tumour probably originated from residual melanocytes with a very low growth rate during 21 years, or which remained quiescent for many years before entering an active growth phase. Exenteration of the orbit results in a maximal nasal clearance of $2 \mathrm{~mm}$ or less, which may have been insufficient totally to eradicate the medially located melanoma. The incomplete removal of the posterior orbital structures by the modified anterior exenteration (Fig 3A) could also account for the local recurrence, but the anterior location of the recurrent neoplasm argues against this.

This case also differs from the other cases in our series in that there was a connection between the nasal and orbital elements of the disease through the nasolacrimal duct without invading mucosa or bone. Therefore, the nasal melanoma is more likely to represent a direct extension of the orbital recurrence than a new nasal focus.

It is of interest that in all eight cases of nasal and conjunctival malignant melanoma the initial tumour originated from areas of conjunctival primary acquired melanosis (PAM) with atypia. This multicentric precursor was noted in approximately $60 \%$ of our patients with invasive conjunctival malignant melanoma and may pose a higher risk of recurrent disease to the patient. Another risk factor of nasal metastasis may be the location of the tumour. Seven out of eight cases showed involvement of the medial ocular structures, including the medial tarsal or bulbar conjunctiva, the plica and the caruncle.
Symptoms of a nasal location of malignant melanoma are recurrent epistaxis or chronic obstruction of the ipsilateral nasal passage, and the clinician should be aware of those symptoms in patients with conjunctival melanomas, especially those that originated from PAM with atypia.

Robertson et $a l^{\prime}$ proposed that malignant melanomas of the nasal cavity and the paranasal sinuses could evolve from either direct extension along vessels or regional metastases via the blood stream, and they thought it would be less probable that the metastases were caused by dissemination of tumour cells to the nasal cavity through tears. However, Weiss et $a l^{2}$ recently found exfoliated atypical melanocytes in the tear film of a patient with multifocal conjunctival melanoma. This finding demonstrates that shedding of melanoma cells into the tear drainage apparatus does occur, but there has been no evidence that these cells have the propensity to cause metastases. Our case has shown that direct' extension through the nasolacrimal duct is another distinct possibility. Such a mode of spread was also observed in another case of conjunctival melanoma. ${ }^{3}$ On histological examination of the exenteration specimen we detected atypical melanocytes in the lumen of the lacrimal apparatus (Fig 5). Serial sectioning showed these cells formed part of a stalactite-like extension from the conjunctival tumour.

Punctal occlusion has been suggested to prevent seeding of malignant cells into the lacrimal apparatus, ${ }^{2}$ but it would not prevent settling of blood-borne metastases or tumour extension along vessels. Surgical procedures may induce dispersion of melanocytes or may not eradicate all the neoplasm. If multicentric conjunctival melanomas, especially those involving the medial canthus, require orbital exenteration, postoperative external beam radiotherapy ${ }^{+}$with megavoltage photons to the socket and nasolacrimal duct may prevent local metastases in the nasal cavity and paranasal sinuses, a condition which is known to be associated with a poor prognosis. ${ }^{15}$

The suggestions of Dr I F Moseley, Department of Radiology, Moorfields Eye Hospital, and Dr M Charlesworth, Department of Radiology, St Bartholomew's Hospital, are gratefully acknowledged. We would like to thank Dr C Wells, Department of Pathology, St Bartholomew's Hospital, for kindly providing the histological sections.

1 Robertson DM, Hungerford JL, McCartney ACE. Malignant melanomas of the conjunctiva, nasal cavity, and paranasal melanomas of the conjunctiva, nasal cavity, and paranasal
sinuses. Am $\mathscr{f}$ Ophthalmol 1989; 108: $440-2$. sinuses. Am $f$ Ophthalmol 1989, 108: 440-2.

Weiss JS, Perusse P, Reale F. Tear cytology in conjunctival melanoma. Am F Ophthalmol 1991; 111: 648-92.

3 Paridaens ADA, McCartney ACE, Curling OM, Lyons CJ, Hungerford JL. Impression cytology of conjunctival melanosis and melanoma. Br $\mathcal{F}$ Ophthalmol 1992; 76: 198201 .

4 Hykin PG, McCartney ACE, Plowman PN, Hungerford JL. Postenucleation orbital radiotherapy for the treatment of malignant melanoma of the choroid with extrascleral extension. BrF Ophthalmol 1990; 74: 36-9.

5 Lund V. Malignant melanoma of the nasal cavity and paranasal sinuses. F Laryngol Otol 1982; 96: 347. 